\begin{tabular}{|c|c|c|c|c|c|}
\hline Revista Clío América & ISSN: 1909-941X & Vol. 11 & No. 21 & enero - junio de 2017 & 48 - 58 \\
\hline \multicolumn{6}{|c|}{ D0I: http://dx.doi.org/10.21676/23897848.2114 } \\
\hline
\end{tabular}

\title{
Estudio exploratorio sobre factores de éxito y barreras para la gestión de conocimiento en organizaciones desarrolladoras de software en Colombia
}

\author{
Exploring success factors and barriers for implementing knowledge management in software \\ development organizations in Colombia
}

RESUMEN: La Gestión del Conocimiento (GC) es una disciplina clave para las Organizaciones Desarrolladoras de Software (ODS), dado que el conocimiento en sus diversas formas es el activo y el producto principal de estas organizaciones. Este artículo identifica los factores de éxito en la implementación de la GC y las barreras que dificultan su aplicación en ODS. Para alcanzar el objetivo se realizó una encuesta entre los representantes de 129 ODS en Colombia. Entre las conclusiones se destacan la identificación de los factores humanos como los más relevantes para el éxito en la GC, y el alto costo y baja disponibilidad de recursos financieros y humanos, y el desconocimiento y la inexperiencia en $\mathrm{GC}$ como los principales obstáculos para la aplicación de la GC. Este estudio podría ser utilizado como una referencia para la identificación y el establecimiento de las condiciones que podrían influir en presentes o futuras implementaciones de GC.

Palabras clave: Barreras; Gestión del Conocimiento; Factores de éxito; Organizaciones Desarrolladoras de Software.

JEL: M1, L2

ABSTRACT: Knowledge Management (KM) is a key discipline for Software Development Organizations (SDO) because the main asset and the main product of these organizations is knowledge in diverse forms. This study identified the factors considered determinants for the success in KM implementation and the barriers that difficult the implementation of KM. For reaching the objective, a survey among representatives of 129 SD0 in Colombia was conducted. One of the findings of this study is the identification of Human-oriented factors as the most relevant factors for the success of KM, and the high cost and low availability of financial and human resources, and unawareness and inexperience in $\mathrm{KM}$ as the main barriers for the implementation of KM. This study could be used as a reference for identifying and establishing the existing conditions that could influence in present or future KM implementations.

Keywords: Barriers; Knowledge Management; Success Factors; Software Development Organizations.

(⿸丆口

Ernesto Amaru Galvis-Lista

Doctor en Ingeniería. Profesor asociado de la Universidad del Magdalena,

Santa Marta - Colombia.

Email:egalvis@unimagdalena.edu.co ORCID: 0000-0002-6012-668X

\section{Mayda Patricia González-Zabala}

Doctora en Ingeniería. Profesora asociada de la Universidad del Magdalena.

Santa Marta - Colombia. Email:mpgonzalez@unimagdalena.edu.co ORCID: 0000-0002-6847-7919

Jenny Marcela Sánchez-Torres

Doctora en Economía de la Innovación. Profesora titular de la Universidad Nacional de Colombia. Bogotá - Colombia. Email: jmsanchezt@unal.edu.co ORCID: 0000-0001-5284-836X

Tipología: Artículo de investigación científica y tecnológica

Fecha de recibido: diciembre 12 de 2016 Fecha de aceptación: abril 19 de 2017

Publicado en línea: abril 28 de 2017

Para citar este artículo: Galvis-Lista, E.A., González-Zabala, M.P., \& Sánchez-Torres, J.M. (2017). Estudio exploratorio sobre factores de éxito y barreras para la gestión de conocimiento en organizaciones desarrolladoras de software en Colombia. Clío América, 11(21), 48 - 58. Doi: http:// dx.doi.org/10.21676/23897848.2114 


\section{INTRODUCCIÓN}

La gestión de conocimiento (GC) es un enfoque para crear 0 agregar valor a partir del aprovechamiento del know-how, la experiencia y los juicios que existen dentro y fuera de la organización (Ruggles, 1998). Este enfoque se centra en ocho categorías de actividades: (1) generación de conocimiento, (2) acceso al conocimiento disponible en fuentes externas, (3) uso de conocimiento en la toma de decisiones, (4) integración de conocimiento en procesos, productos y servicios; (5) codificación de conocimiento en documentos, bases de datos y software; (6) promoción de la creación del conocimiento a través de la cultura y los incentivos, (7) transferencia del conocimiento existente a otras partes de la organización y (8) evaluación de activos de conocimiento y del impacto de la GC.

En Colombia su implementación en organizaciones desarrolladoras de software (ODS) ha sido estudiada y reportada en publicaciones científicas desde los últimos diez años. Los principales aspectos investigados han sido la aplicación de la GC en la mejora de procesos de software y el mantenimiento de software (Bravo \& Puerto, 2012; Capote, Llantén, Pardo, \& Collazos, 2009; Capote, Llantén, Pardo, Gonzalez, \& Collazos, 2008; Castro, García, \& Crespo, 2013; García, Amescua, Sánchez, \& Bermón, 2011). Sin embargo, no han sido reportados resultados empíricos sobre los factores de éxito para la implementación efectiva de la GC en las ODS en Colombia.

La implementación efectiva de la GC en las organizaciones está sujeta a ciertos elementos que aseguran su eficacia y sostenibilidad. Estos elementos son conocidos como factores habilitadores o factores de éxito de la GC, los cuales pueden ser entendidos como mecanismos organizacionales para, intencional y consistentemente, desarrollar conocimiento (Heeseok Lee \& Byounggu Choi, 2003). Estos factores tienen una fuerte influencia en el desempeño de la organización en la GC y en el desempeño y eficiencia de la organización en general (Allameh, Zare, \& Davoodi, 2011; Bhatti, Hussain, \& Iqbal, 2013; Heeseok Lee \& Byounggu Choi, 2003; C.-F. Ho, Hsieh, \& Hung, 2014; C.-T. Ho, 2009; Yeh, Lai, \& Ho, 2006).
Dichos factores de éxito han sido estudiados por varios autores, tales como: De Freitas, y Yaber, G. (2015); Liophanich (2014); Domínguez y Martins (2014); AL-Hakim y Hassan (2012); Valmohammadi (2010); y Heisig (2009), por solo mencionar algunos. De estos trabajos se destaca el realizado por Heisig (2009) quien desarrolló la armonización de 160 marcos de trabajo (Frameworks) de GC propuestos desde la industria, la academia, y las organizaciones dedicadas a la generación de estándares. Este trabajo es uno de los más citados y se ha constituido en un referente en el tema.

De acuerdo con Heisig (2009) los factores de éxito de la GC se pueden agrupar en cuatro categorías principales y nueve subcategorías. Las categorías propuestas son: factores humanos, organización, tecnología y proceso de gestión; y las nueve subcategorías son: cultura organizacional, conocimiento de las personas y motivación, liderazgo y apoyo de la dirección, procesos organizacionales, estructura organizacional, infraestructura de tecnología de información, aplicaciones y herramientas, estrategia organizacional, y medición y evaluación. En la Tabla 1 se presenta una síntesis de la relación de las categorías y subcategorías, así como los términos identificados por Heisig (2009) en su estudio. Cabe destacar que la publicación de Heisig es uno de los trabajos con alto número de citaciones en Scopus (171), ISI Web of Knowledge (113) y Google Scholar (347), lo cual evidencia su pertinencia y vigencia para fundamentar el presente estudio exploratorio.

Es importante destacar que en el contexto particular de las ODS, la GC y sus factores de éxito han sido concebidos como elementos claves pues el principal activo y también el principal producto de estas organizaciones es el conocimiento en diversas formas (Aurum, Daneshgar, \& Ward, 2008; Bjørnson \& Dingsøyr, 2008; Dingsøyr, Bjornson, \& Shull, 2009; Rus \& Lindvall, 2002). En este orden de ideas, el propósito de este artículo es presentar los resultados de una investigación que busca responder a la pregunta: ¿cuáles son los factores de éxito y las barreras que inciden en la implementación de procesos de GC en las ODS colombianas? Esta investigación de carácter exploratorio brinda evidencia empírica inicial sobre la implementación de la GC en ODS en Colombia. 


\section{Tabla 1.}

\section{Factores de éxito de la GC}

\begin{tabular}{|c|c|c|}
\hline CATEGORÍA & SUBCATEGORÍAS & TÉRMINOS RELACIONADOS \\
\hline \multirow{3}{*}{ 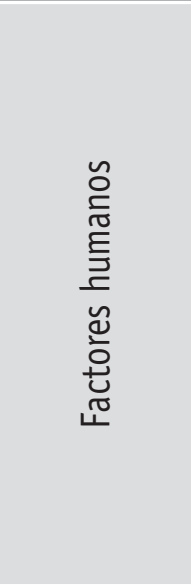 } & Cultura organizacional & $\begin{array}{l}\text { Cultura corporativa, cultura del conocimiento, conocimiento } \\
\text { orientado a la cultura, cultura de compartir el conocimiento, } \\
\text { cultura y poder, cultura de aprendizaje, factores culturales y } \\
\text { sociales, sistema de valores, valores y normas, valores. }\end{array}$ \\
\hline & $\begin{array}{l}\text { Conocimiento de las } \\
\text { personas y motivación }\end{array}$ & $\begin{array}{l}\text { Empleados, personal, habilidades, habilidades de los em- } \\
\text { pleados, conocimiento y experiencia de los empleados, } \\
\text { características personales, conocimiento personal, capacidades } \\
\text { de conocimiento personal, humano, recursos humanos, motiva- } \\
\text { ción y cualificación. }\end{array}$ \\
\hline & $\begin{array}{l}\text { Liderazgo y apoyo de } \\
\text { la dirección }\end{array}$ & $\begin{array}{l}\text { Liderazgo del conocimiento, liderazgo y apoyo, apoyo de la } \\
\text { dirección, apoyo de la alta gerencia, conocimiento orientado a } \\
\text { la gestión, gestión. }\end{array}$ \\
\hline \multirow{2}{*}{ 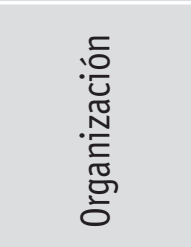 } & $\begin{array}{l}\text { Procesos } \\
\text { organizacionales }\end{array}$ & $\begin{array}{l}\text { Procesos organizacionales, organización de procesos, procesos } \\
\text { de negocio. }\end{array}$ \\
\hline & $\begin{array}{l}\text { Estructura } \\
\text { organizacional }\end{array}$ & $\begin{array}{l}\text { Estructura organizacional, diseño organizacional, infraestructu- } \\
\text { ra organizacional. }\end{array}$ \\
\hline \multirow{2}{*}{ 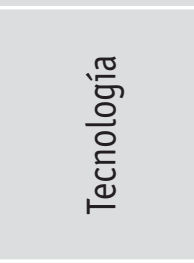 } & $\begin{array}{l}\text { Infraestructura } \\
\text { de Tecnología de } \\
\text { Información - TI }\end{array}$ & $\begin{array}{l}\text { Tecnología de información y las comunicaciones, infraestructu- } \\
\text { ra tecnológica. }\end{array}$ \\
\hline & $\begin{array}{l}\text { Aplicaciones y } \\
\text { herramientas }\end{array}$ & Sistemas tecnológicos, tecnologías de GC \\
\hline \multirow{2}{*}{ 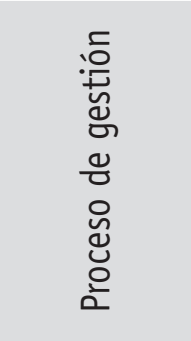 } & $\begin{array}{l}\text { Estrategia } \\
\text { organizacional }\end{array}$ & $\begin{array}{l}\text { Objetivos, objetivos organizacionales, objetivos concretos y } \\
\text { medibles, visión, estrategia basada en conocimiento, compor- } \\
\text { tamiento estratégico, misión, visión a largo, mediano y corto } \\
\text { plazo, política, planeación. }\end{array}$ \\
\hline & Medición y evaluación & $\begin{array}{l}\text { Control de conocimientos, control de conocimientos y aprendi- } \\
\text { zaje, métrica, criterios de medición, medición del rendimiento } \\
\text { de la GC, indicadores de desempeño. }\end{array}$ \\
\hline
\end{tabular}

Fuente: Elaboración propia basada en (Heisig, 2009)

\section{METODOLOGÍA}

Para el estudio exploratorio se utilizó una metodología compuesta por tres etapas: definición de la población en estudio, recolección de datos y análisis de resultados. En las siguientes subsecciones se describe brevemente el diseño metodológico de este estudio exploratorio.

\section{Definición de la Población en Estudio}

La población objetivo del estudio, es decir el universo a estudiar, son las ODS colombianas. Para su identificación se consultó la información disponible en los portales web de FedeSoft (2014), ParqueSoft (2014), ESI Center (2015) actualmente Fundación Tecnalia Colombia, y la Guía de Soluciones TIC de la 
planta empresa Datamarket Solutions SAS (2014). Luego se procedió a buscar los datos de contacto de personas con cargos directivos en los sitios web de las ODS identificadas y en varios motores de búsqueda de uso general. Para los casos en los que no se encontró el dato de contacto de una persona, se registró la dirección de contacto de información corporativa o la de servicio al cliente. La actividad permitió identificar 884 contactos de personas con cargos en niveles gerenciales en 475 ODS. Es importante destacar que de acuerdo con el censo realizado por FEDESOFT se tenían 1813 empresas del sector TIC oficialmente registradas en las Cámaras de Comercio del país. En este universo, FEDESOFT identificó 765 empresas de software enfocadas principalmente en el desarrollo software y otras actividades como consultoría o servicios de software (Fedesoft, 2012, p. 52).

\section{Recopilación de Datos}

Para la obtención de datos se diseñó una encuesta compuesta de dos secciones. La primera sección se enfocó en la recopilación de datos demográficos de los encuestados y las organizaciones. La segunda sección se enfocó en clasificar los factores de éxito de la GC, descritos en la Tabla 1, utilizando la escala de evaluación de tres categorías: totalmente determinante, parcialmente determinante y no determinante para la implementación exitosa de la GC. Además, se preguntó por cinco barreras que obstaculizan la implementación de GC en las ODS, ordenadas de mayor a menor grado de impacto para la organización.

La encuesta se realizó utilizando un cuestionario en línea configurado en la herramienta Limesurvey, y se aplicó a un responsable por ODS, para un total de 475 ODS invitadas a responder la encuesta. Vale la pena aclarar que, aunque se enviaron invitaciones a varios miembros de cada organización, se indicó que la encuesta debía ser diligenciada por el responsable de los procesos de GC o por un administrador de alto nivel. La encuesta se realizó en un periodo de cuatro meses.

De las 475 ODS potenciales para el estudio, se obtuvo respuesta de 122 , es decir, del $25,7 \%$ de las organizaciones invitadas. Es importante destacar que la tasa bruta de respuesta está dentro de los rangos de tasas de respuesta en investigación organizacional identificadas por Baruch y Holtom (Baruch \& Holtom, 2008). En relación con los datos de FEDESOFT, se obtuvo respuesta del 15,9\% de las ODS registradas en cámaras de comercio en Colombia. De acuerdo con la ubicación geográfica de las ODS encuestadas, el $85 \%$ se ubicaba en las tres principales ciudades en el país. Bogotá (67), Cali (21) y Medellín (16), el $15 \%$ restante se distribuye en 11 ciudades capitales de departamentos del país, concentradas principalmente en la región andina, exceptuando la ciudad de Cartagena ubicada en la región Caribe. En la Tabla 2 se puede apreciar la distribución por ubicación geográfica de las ODS consultadas para el estudio.

Respecto al tamaño de las organizaciones, en términos de número de empleados, se encontró que el $36,1 \%$ corresponde a pequeña empresa ( $\mathrm{N}=44)$, el $29,5 \%$ a micro empresa $(\mathrm{N}=36)$, el $18 \%$ a mediana $(\mathrm{N}=22)$ y el $16,46 \%$ a empresa grande $(\mathrm{N}=20)$. En términos de la principal actividad se encontró que el $80,3 \%$ eran organizaciones desarrolladoras de software, y el restante $19,7 \%$ de las empresas se dedica a otras actividades relacionadas con desarrollo de software, tales como consultoría $(6,6 \%)$, integración de sistemas $(4,9 \%)$, outsourcing de aplicaciones $(4,9 \%)$ o mantenimiento de software $(3,3$ $\%)$. En cuanto al enfoque de desarrollo que emplean las empresas se detectó que el $58,2 \%(\mathrm{~N}=71)$ de las ODS encuestadas utiliza un enfoque ágil y el 41,8 \% $(\mathrm{N}=51)$ utiliza un enfoque tradicional. También se detectó que el 78,7 \% de las empresas tiene algún tipo de certificación para sus procesos, tales como ISO 9001, CMMI, IT Mark u otras. 


\section{Tabla 2.}

Datos demográficos de las ODS consultadas

\begin{tabular}{|l|c|c|c|}
\hline \multicolumn{1}{|c|}{ CIUDAD } & FRECUENCIA & $\%$ & \% ACUMULADO \\
\hline Bogotá & 67 & 54,9 & 54,9 \\
\hline Cali & 21 & 17,2 & 72,1 \\
\hline Medellín & 16 & 13,1 & 85,2 \\
\hline Bucaramanga & 5 & 4,1 & 89,3 \\
\hline Manizales & 3 & 2,5 & 91,8 \\
\hline Armenia & 2 & 1,6 & 93,4 \\
\hline Cartagena & 2 & 1,6 & 95,1 \\
\hline Pasto & 2 & 1,6 & 96,7 \\
\hline Barranquilla & 1 & 0,8 & 97,5 \\
\hline Ibagué & 1 & 0,8 & 98,4 \\
\hline Pereira & 1 & 0,8 & 99,2 \\
\hline Popayán & 1 & 0,8 & 100,0 \\
\hline Total & 122 & 100,0 & \\
\hline
\end{tabular}

Fuente: Elaboración propia

En relación con el perfil de las personas encuestadas, manifestó tener más de seis años de vinculación a la el 78,7 \% expresó tener más de seis años de expe- organización, en tanto que solo el 4,1 \% reportó que riencia en el sector software, de los cuales el 69,7\% su tiempo de vinculación era menor a un año (Tabla 3).

\section{Tabla 3.}

Tiempo de vinculación del personal encuestado

\begin{tabular}{|l|c|c|c|c|}
\multicolumn{1}{|c|}{ TIEMPO } & $\begin{array}{c}\text { EXPERIENCIA EN DESARROLLO } \\
\text { SOFTWARE }\end{array}$ & \multicolumn{2}{c|}{ EXPERIENCIA EN LA EMPRESA } \\
\hline & $\mathrm{N}$ & $(\%)$ & $\mathrm{N}$ & $(\%)$ \\
\hline Más de 10 años & 79 & 64,8 & 61 & 50 \\
\hline 6 a 10 años & 17 & 13,9 & 24 & 19,7 \\
\hline 1 a 5 años & 13 & 10,7 & 32 & 26,2 \\
\hline Menos de 1 año & 13 & 10,7 & 5 & 4,1 \\
\hline Total & & 129 & & 100,0 \\
\hline
\end{tabular}

Fuente: Elaboración propia 


\section{Procesamiento de los Datos y Análisis de Resultados}

El procesamiento de los datos consistió en un análisis cuantitativo, específicamente de estadística descriptiva en las dos primeras preguntas de investigación, obteniendo tablas de frecuencia basadas en variables ordinales y en distribuciones multivariadas. Para organizar los resultados y la discusión se tomó como referente la clasificación de factores de éxito que propone Heisig (2009). Además, para el análisis de los factores de éxito para incorporación de procesos de GC identificados por las ODS en relación con el tamaño de la empresa, la orientación metodológica o la certificación de procesos, se procedió a calcular el coeficiente Tau $b$ de Kendall.

\section{RESULTADOS}

Los resultados obtenidos evidenciaron que los factores asociados a la categoría de factores humanos fueron considerados como totalmente relevantes. Específicamente, el factor de cultura organizacional fue evaluado como totalmente determinante por el $73 \%$ de las ODS, el de conocimiento de las personas y motivación por el $68 \%$ de las ODS y el de liderazgo y apoyo de la dirección por el 69,7\%. El factor de estrategia organizacional, de la categoría gestión de procesos, también fue considerado como totalmente determinante por el 65,5\% de las ODS encuestadas. Es importante decir que resultados similares fueron identificados en otras investigaciones relacionadas con la implementación de procesos asociados a la GC (Marulanda, López \& López, 2016; De Freitas \& Yaber
2015; Salazar, 2014), también cabe destacar que el factor humano y el factor de cultura organizacional han sido considerados como relevantes, no solo para la gestión del conocimiento, sino para el éxito y proyección de las organizaciones (Segrera, Daza \& Labarcés, 2014; Pedraza, Socarras, Jiménez \& Romero, 2014; Viloria, Pedraza, Cuesta \& Pérez, 2016).

En contraposición, los factores con más bajo puntaje en la calificación de totalmente determinante fueron los de infraestructura de tecnologías de la información, aplicaciones y herramientas, medición y evaluación, y estrategia organizacional, consideradas como totalmente determinante por el $41,8 \%, 41 \%, 40,2$ $\%$ y $34,4 \%$ de la ODS, respectivamente. En la Tabla 4 se presenta la evaluación de los factores de éxito analizados distribuidos por categorías de evaluación.

Por otra parte, se calculó el coeficiente Tau b de Kendall para el análisis de los factores de éxito para incorporación de procesos de GC identificados por las ODS en relación con el tamaño de la empresa, la orientación metodológica o la certificación de procesos. El cálculo del coeficiente y su significancia permitieron apreciar que se encuentra una asociación entre el factor de éxito "cultura organizacional" y el tamaño de la organización (coeficiente 0,224$)$ y con la certificación de procesos (coeficiente 0,235 ). Para los otros factores de éxito en estudio no se encontró asociación directa con las características de las ODS consideradas en el estudio. En la Tabla 5 se presenta el valor obtenido para el coeficiente para cada factor de éxito y las características en estudio.

\section{Tabla 4.}

Evaluación de los factores de éxito por parte de las ODS

\begin{tabular}{|c|c|c|c|c|c|c|}
\hline \multirow{2}{*}{ FACTOR DE ÉXITO } & \multicolumn{2}{|c|}{$\begin{array}{c}\text { NO ES } \\
\text { DETERMINANTE }\end{array}$} & \multicolumn{2}{|c|}{$\begin{array}{l}\text { PARCIALMENTE } \\
\text { DETERMINANTE }\end{array}$} & \multicolumn{2}{|c|}{$\begin{array}{c}\text { TOTALMENTE } \\
\text { DETERMINANTE }\end{array}$} \\
\hline & $\mathrm{N}$ & $\%$ & $\mathbf{N}$ & $\%$ & $\mathbf{N}$ & $\%$ \\
\hline Cultura organizacional & 4 & 3,3 & 29 & 23,8 & 89 & 73,0 \\
\hline Liderazgo y apoyo de la dirección & 4 & 3,3 & 33 & 27,0 & 85 & 69,7 \\
\hline Conocimientos y motivación de las personas & 3 & 2,5 & 36 & 29,5 & 83 & 68,0 \\
\hline Estrategia organizacional & 3 & 2,5 & 39 & 32,0 & 80 & 65,6 \\
\hline
\end{tabular}


Estudio exploratorio sobre factores de éxito y barreras para la gestión de conocimiento en organizaciones desarrolladoras de software en Colombia

\begin{tabular}{|c|c|c|c|c|c|c|}
\hline \multirow[t]{2}{*}{ FACTOR DE ÉXITO } & \multicolumn{2}{|c|}{$\begin{array}{c}\text { NO ES } \\
\text { DETERMINANTE }\end{array}$} & \multicolumn{2}{|c|}{$\begin{array}{l}\text { PARCIALMENTE } \\
\text { DETERMINANTE }\end{array}$} & \multicolumn{2}{|c|}{$\begin{array}{c}\text { TOTALMENTE } \\
\text { DETERMINANTE }\end{array}$} \\
\hline & $\mathrm{N}$ & $\%$ & $\mathrm{~N}$ & $\%$ & $\mathrm{~N}$ & $\%$ \\
\hline Procesos organizacionales & 12 & 9,8 & 48 & 39,3 & 62 & 50,8 \\
\hline Medición y evaluación & 14 & 11,5 & 59 & 48,4 & 49 & 40,2 \\
\hline Infraestructura tecnológica & 10 & 8,2 & 61 & 50,0 & 51 & 41,8 \\
\hline Aplicaciones y herramientas & 12 & 9,8 & 60 & 49,2 & 50 & 41,0 \\
\hline Estructura organizacional & 22 & 18,0 & 58 & 47,5 & 42 & 34,4 \\
\hline
\end{tabular}

Fuente: Elaboración propia

Tabla 5.

Asociación entre los factores de éxito y las características de las empresas

\begin{tabular}{|c|c|c|c|c|}
\hline FACTOR DE ÉXITO & MEDIDA & TAMAÑO & $\begin{array}{l}\text { ORIENTACIÓN } \\
\text { METODOLÓGICA }\end{array}$ & $\begin{array}{l}\text { CERTIFICACIÓN } \\
\text { DE PROCESOS }\end{array}$ \\
\hline \multirow{2}{*}{ Cultura organizacional } & Coeficiente & $0,224^{* *}$ & $-0,131$ & $0,235^{* *}$ \\
\hline & Significancia & 0,007 & 0,143 & 0,009 \\
\hline \multirow{2}{*}{$\begin{array}{l}\text { Conocimientos y motivación de las } \\
\text { personas }\end{array}$} & Coeficiente & 0,006 & 0,066 & $-0,071$ \\
\hline & Significancia & 0,943 & 0,46 & 0,431 \\
\hline \multirow{2}{*}{ Liderazgo y apoyo de la dirección } & Coeficiente & $-0,058$ & 0,067 & $-0,081$ \\
\hline & Significancia & 0,481 & 0,456 & 0,363 \\
\hline \multirow{2}{*}{ Procesos organizacionales } & Coeficiente & 0,109 & 0,053 & $-0,003$ \\
\hline & Significancia & 0,177 & 0,544 & 0,977 \\
\hline \multirow{2}{*}{ Estructura organizacional } & Coeficiente & $-0,029$ & 0,01 & $-0,02$ \\
\hline & Significancia & 0,717 & 0,91 & 0,82 \\
\hline \multirow{2}{*}{ Infraestructura tecnológica } & Coeficiente & $-0,221 * *$ & 0,045 & $-0,177^{* *}$ \\
\hline & Significancia & 0,006 & 0,61 & 0,044 \\
\hline \multirow{2}{*}{ Aplicaciones y herramientas } & Coeficiente & $-0,309 * *$ & $-0,039$ & $-0,209 * *$ \\
\hline & Significancia & 0 & 0,653 & 0,017 \\
\hline \multirow{2}{*}{ Estrategia organizacional } & Coeficiente & 0,148 & $-0,01$ & 0,159 \\
\hline & Significancia & 0,074 & 0,913 & 0,077 \\
\hline \multirow{2}{*}{ Medición y evaluación } & Coeficiente & 0,06 & $-0,084$ & $-0,02$ \\
\hline & Significancia & 0,454 & 0,339 & 0,818 \\
\hline
\end{tabular}

Fuente: Elaboración propia 
En lo concerniente a la indagación sobre las principales dificultades que tienen las ODS para implementar procesos de GC, se obtuvieron 324 comentarios por parte de 94 ODS participantes.
Del análisis de los comentarios se obtuvieron 14 categorías de clasificación, las cuales se presentan en la Tabla 6, en donde se relacionan las categorías con los comentarios y el número de participantes.

\section{Tabla 6.}

Categorías de dificultades para implementar procesos de GC en las ODS

\section{CATEGORÍA}

Altos costos y baja disponibilidad de recursos económicos y humanos

Desconocimiento e inexperiencia sobre GC

Formas de pensar, motivación y compromiso de las personas

Insuficiente planificación, priorización, liderazgo y control de la alta dirección

Carencia o uso inadecuado de métodos y herramientas

Cultura organizacional existente

Estructura y procesos organizacionales

Carencia de métricas y procesos de evaluación

Rotación del personal

Cambios en la organización y su entorno

Niveles de formación de las personas

Falta de interacción y apoyo con entidades externas

Los clientes no valoran la implementación de la GC en la organización

Acceso limitado a nuevas fuentes de conocimiento

\section{COMENTARIOS PARTICIPANTES}

50

36

32

31

29

23

18

12

9

7

5

3

5

4

Fuente: Elaboración propia basada en los datos recolectados con la encuesta.

La categoría con el mayor número de comentarios fue "Altos costos y baja disponibilidad de recursos económicos y humanos", seguida de "Desconocimiento e inexperiencia sobre GC" y "Formas de pensar, motivación y compromiso de las personas". En la primera categoría se encontraron comentarios como: "Falta de recursos (económicos, tecnológicos y humanos) para implementar la gestión del conocimiento", "La creación de conocimiento es un lujo que no podemos costear" $\mathrm{y}$ "Para aplicarlo realmente se requiere inversión importante en recursos, herramientas". Así mismo, algunos comentarios clasificados en la segunda categoría fueron: "Falta de consciencia y conocimiento de este tema en los miembros de los proyectos", "Desconocimiento de los beneficios y ventajas de gestionar el conocimiento de la organización.", y "Conocimiento en gestión de conocimiento muy teórico, no se conocen experiencias donde se aplique en su totalidad". Por su parte, para la tercera categoría se encontraron comentarios como: "Sensibilización/ actitud de las personas para abordar los procesos de gestión de conocimiento", "Es difícil convencer a las personas de lo crítico que es la evaluación del conocimiento" y "Falta de motivación para la implementación de GC". 
Estudio exploratorio sobre factores de éxito y barreras para la gestión de conocimiento en organizaciones desarrolladoras de software en Colombia

\section{CONCLUSIÓN}

Los hallazgos relacionados con la pregunta de investigación mostraron un predominio de los factores humanos sobre factores organizativos o tecnológicos, incluso en las organizaciones de desarrollo de tecnología. Específicamente, se encontró que el factor de cultura organizacional fue evaluado como totalmente determinante para la GC por más del 70 $\%$ de las organizaciones en estudio. Siendo, a su vez, el único factor que tiene correlación con el tamaño de la empresa y la certificación de los procesos.

Estas correlaciones pueden explicarse en el sentido que los procesos de certificación implican que los miembros de la organización deben estructurarse y asumir unas dinámicas orientadas a formalizar, ejecutar y evidenciar las actividades organizacionales; siendo estas actividades parte de las actividades requeridas para la $\mathrm{GC}$. En este sentido, se considera que el trabajo futuro se debería orientar hacia la identificación de las características particulares de cultura, personal y el liderazgo que influyen positivamente en la aplicación de GC en ODS en Colombia.

Así mismo, el estudio mostró que los factores de liderazgo y apoyo a la dirección, conocimientos y motivación de las personas y estrategia organizacional fueron identificados como totalmente relevantes por más del $65 \%$ de las ODS. Es decir, la GC se puede viabilizar si desde la dirección se crean las estrategias para este fin, y si los líderes de proceso están comprometidos con las mismas.

Por otra parte, se identificó que la subcategoría "Estructura organizacional" del aspecto organización, tuvo el valor más bajo en el nivel de "totalmente determinante". Este resultado podría ser una consecuencia natural de la creciente tendencia en la industria de software en organizaciones flexibles y ágiles.

En general, se tiene que la Industria Software colombiana es una "tierra fértil" para trabajar en temas de GC en la investigación y la práctica. En este sentido, el análisis sobre la importancia de cada categoría de los factores de éxito de $\mathrm{GC}$ debe ser investigado en detalle, para lo cual sería pertinente realizar estudios de casos que permitan obtener una comprensión más profunda de cada uno de los factores de éxito y cómo ser más eficaz en la aplicación de la GC en ODS colombianas. Además, los resultados podrían ser utilizados como referente para identificar qué factor de éxito es "más importante" para las ODS en Colombia, con el fin de priorizar el trabajo por hacer. Así mismo, es importante incorporar en futuras investigaciones, otras variables y la investigación detallada de cada factor de éxito GC. Además, sería interesante contrastar estos resultados con otros países, como quiera que se espera que los factores de éxito GC serán diferentes entre regiones, países y culturas.

Hay que considerar que este estudio fue exploratorio, y sus resultados y análisis fueron de tipo descriptivo. Sin embargo, es una contribución para la comprensión de la aplicación de los conocimientos en ODS en Colombia, siendo este un primer paso en una iniciativa de investigación en curso, por lo tanto, los hallazgos son útiles y constituyen una línea de base preliminar para la investigación futura.

\section{REFERENCIAS BIBLIOGRÁFICAS}

Allameh, S. M., Zare, S. M., \& Davoodi, S. Mohammad. (2011). Examining the Impact of KM Enablers on Knowledge Management Processes. Procedia Computer Science, 3, 1211-1223. https://doi.org/10.1016/j. procs.2010.12.196

AL-Hakim, L.A.Y., \& Hassan, S. (2012). Critical Success Factors of Knowledge Management, Innovation and Organisational Performance: An Empirical Study of the Iraqi Mobile Telecommunication Sector. British Journal of Economics, Finance and Management Sciences, 4(1) 31-49.

Aurum, A., Daneshgar, F., \& Ward, J. (2008). Investigating Knowledge Management practices in software development organisations - An Australian experience. Information and Software Technology, 50(6), 511533. https://doi.org/10.1016/j.infsof.2007.05.005

Baruch, Y., \& Holtom, B. C. (2008). Survey response rate levels and trends in organizational research. Human Relations, 61(8), 1139-1160. https://doi. org/10.1177/0018726708094863

Bhatti, W. A., Hussain, N., \& Iqbal, J. (2013). Leadership role in integrating knowledge management enablers. World Applied Sciences Journal, 26(1), 55-60. https:// doi.org/10.5829/idosi.wasj.2013.26.01.2453 
Bjørnson, F. 0., \& Dingsøyr, T. (2008). Knowledge management in software engineering: A systematic review of studied concepts, findings and research methods used. Information and Software Technology, 50(11), 1055-1068. https://doi.org/10.1016/j. infsof.2008.03.006

Bravo, G., \& Puerto, A. (2012). ELLES-Enterprise lessons learned system. En Computing Congress (CCC), 2012 7th Colombian (1-6). https://doi.org/10.1109/ ColombianCC.2012.6398006

Capote, J., Llantén, C. J., Pardo, C., \& Collazos, C. (2009). Knowledge management in a software process improvement program in micro, small and medium-sized enterprises: KMSPI Model. Revista Facultad de Ingenieria, (50), 205-216.

Capote, J., Llantén, C. J., Pardo, C., González, A., \& Collazos, C. (2008). Gestión del conocimiento como apoyo para la mejora de procesos software en las micro, pequeñas y medianas empresas. Ingenieria $e$ investigacion, 28.

Castro, S. J. B., García, V. H. M. \& Crespo, R. G. (2013). Emerging Concepts between Software Engineering and Knowledge Management. En L. Uden, F. Herrera, J. B. Pérez, \& J. M. C. Rodríguez (Eds.), 7th International Conference on Knowledge Management in Organizations: Service and Cloud Computing (13-24). Springer Berlin Heidelberg. Recuperado de http://link.springer.com/ chapter/10.1007/978-3-642-30867-3_2

Datamarket Solutions SAS. (2014). Proveedores y Empresas de Software en Colombia - Guía Soluciones TIC. Recuperado de http://www.guiadesolucionestic. com/component/sobi2/?catid=3

De Freitas, V., \& Yaber, G. (2015). Una Taxonomía de los Factores Clave de Éxito en la Implantación de Sistemas de Gestión del Conocimiento en Instituciones de Educación Superior. GECONTEC: Revista Internacional de Gestión del Conocimiento y la Tecnología, 3 (1), 69- 86.

Dingsøyr, T., Bjornson, F. 0., \& Shull, F. (2009). What Do We Know about Knowledge Management? Practical Implications for Software Engineering. Software, IEEE, 26 (3), 100-103.

Dominguez, R.V., \& Martins, M. F. (2014). Knowledge Management: an Analysis From the Organizational Development. Journal of Technology Management \& Innovation, 9(1), 131-147.

ESI Center SINERTIC Andino. (2015). ESICenter Sinertic. Recuperado el 30 de enero de 2015, a partir de http:// www.esicenter-sinertic.org/index.php/quienes-somos
Fedesoft. (2012). Estudio de la Caracterización de Productos y Servicios de la Industria de Software y Servicios Asociados 2012. Bogotá, Colombia": Federación Colombiana de la Industria del Software. Recuperado de http://www.fedesoft.org/Downloads/ EstudiocifrassectorSW2012.pdf

Fedesoft. (2014). Catálogo de Empresas Fedesoft | Fedesoft | Federación Colombiana de la Industria de Software y TI. Recuperado de http://fedesoft.org/ catalogo-de-empresas/

Fundación PARQUESOFT. (2014). Empresas - PARQUESOFT. Recuperado de http://parquesoft.com/index. php?option=com_content $\&$ view=category\&layout=b log\&id=31\&Itemid $=139$

García, J., Amescua, A., Sánchez, M. I., \& Bermón, L. (2011). Design guidelines for software processes knowledge repository development. Information and Software Technology, 53(8), 834-850. https://doi. org/10.1016/j.infsof.2011.03.002

Hassan, H.M. Lotfollah, f. \& Negar, M. (2012). Comprehensive Model of Business Intelligence: A Case Study of Nano's Companies. Indian Journal of Science and Technology, 5(6), 2851-2859.

Heeseok, L., \& Byounggu Choi. (2003). Knowledge Management Enablers, Processes, and Organizational Performance: An Integrative View and Empirical Examination. Journal of Management Information Systems, 20(1), 179-228.

Heisig, P. (2009). Harmonisation of Knowledge Management - Comparing 160 KM Frameworks Around the Globe. Journal of Knowledge Management, 13(4), 4-31. https://doi.org/10.1108/13673270910971798

Ho, C.F., Hsieh, P.H., \& Hung, W. H. (2014). Enablers and processes for effective knowledge management. Industrial Management \& Data Systems, 114(5), 734754. https://doi.org/10.1108/IMDS-08-2013-0343

Ho, C. T. (2009). The relationship between knowledge management enablers and performance. Industrial Management \& Data Systems, 109(1), 98-117. https://doi.org/10.1108/02635570910926618

Liophanich, C. (2014). An Investigation of Knowledge Management Implementation: Multiple Case Study in Mobile Telecommunication Industry. Journal of Industrial and Intelligent Information, 2(2), 159-163.

Marulanda, C., López, M., \& LópezLópez, F. (2016). La Cultura Organizacional y las Competencias para la Gestión del Conocimiento en las Pequeñas y Medianas Empresas (PYMEs) de Colombia. Información tecnológica. 27(6) http://dx.doi.org/10.4067/ S0718-07642016000600002 
Estudio exploratorio sobre factores de éxito y barreras para la gestión de conocimiento en organizaciones desarrolladoras de software en Colombia

Pedraza, A. L., Socarrás, P. X., Jiménez, V. M., \& Romero, R. I. (2014). Estudio del clima organizacional en una empresa prestadora de servicios en salud ocupacional de la ciudad de Santa Marta, Colombia. Clío América, $8(15), 47-74$

Ortíz, S. J., Daza, C. A. \& Labarcés, B. C. (2014). La cultura organizacional de los operadores turísticos de Santa Marta 2012 - 2013. Clío América, 8 (15), 22 - 35

Ruggles, R. (1998). The state of the notion: Knowledge management in practice. California Management Review, (3), 80-89.

Rus, I., \& Lindvall, M. (2002). Guest Editors' Introduction: Knowledge Management in Software Engineering. IEEE Software, 19(3), 26-38.

Salazar, F. (2014). Estrategias para la implementación de Gestión del Conocimiento para la empresa UNETelefónica de Pereira. Proyecto de Grado. Universidad Nacional de Colombia. Maestría en Administración. Manizales, Colombia.
Yeh, Y. J., Lai, S. Q., \& Ho, C.T. (2006). Knowledge management enablers: a case study. Industrial Management \& Data Systems, 106(6), 793-810. https://doi. org/10.1108/02635570610671489

Valmohammadi, C. (2010). Identification and Priorization of Critical Success Factors of Knowledge Management in Iranian SMEs: An Experts' View. African Journal of Business Management, 4(6): 915-924.

Viloria, E. J, Pedraza, A. L, Cuesta, T. K., \& Pérez, C. K. (2016). Liderazgo informal en las organizaciones: reflexiones sobre su impacto e influencia en la competitividad. Clío América, 10 (19), 31 - 42 